\title{
Working memory training revisited: A multi-level meta-analysis of n-back training studies
}

\author{
Anna Soveri $^{1} \cdot$ Jan Antfolk $^{1} \cdot$ Linda Karlsson $^{1} \cdot$ Benny Salo $^{1} \cdot$ Matti Laine $^{1,2}$
}

Published online: 23 January 2017

(C) Psychonomic Society, Inc. 2017

\begin{abstract}
The efficacy of working memory (WM) training has been a controversial and hotly debated issue during the past years. Despite a large number of training studies and several meta-analyses, the matter has not yet been solved. We conducted a multi-level meta-analysis on the cognitive transfer effects in healthy adults who have been administered WM updating training with n-back tasks, the most common experimental WM training paradigm. Thanks to this methodological approach that has not been employed in previous meta-analyses in this field, we were able to include effect sizes from all relevant tasks used in the original studies. Altogether 203 effect sizes were derived from 33 published, randomized, controlled trials. In contrast to earlier meta-analyses, we separated task-specific transfer (here untrained n-back tasks) from other WM transfer tasks. Two additional cognitive domains of transfer that we analyzed consisted of fluid intelligence (Gf) and cognitive control tasks. A medium-sized transfer effect was observed to untrained n-back tasks. For other WM tasks, Gf, and cognitive control, the effect sizes were of similar size and very small. Moderator analyses showed no effects of age, training dose, training type (single vs. dual), or WM and Gf transfer task contents (verbal vs. visuospatial). We conclude that a substantial part of transfer following WM training with
\end{abstract}

Electronic supplementary material The online version of this article (doi:10.3758/s13423-016-1217-0) contains supplementary material, which is available to authorized users.

Anna Soveri

anna.soveri@abo.fi

1 Department of Psychology, Åbo Akademi University, Turku, Finland

2 Turku Brain and Mind Center, University of Turku, Turku, Finland the n-back task is task-specific and discuss the implications of the results to WM training research.

Keywords Cognitive training · Working memory · Executive functions $\cdot \mathrm{N}$-back $\cdot$ Meta-analysis

Working memory (WM) training has stirred considerable interest amongst researchers and public at large during the past decade (von Bastian \& Oberauer, 2014; Green \& Bavelier, 2008; Klingberg, 2010; Lövdén, Bäckman, Lindenberger, Schaefer, \& Schmiedek, 2010; Morrison \& Chein, 2011). The main reason for this widespread interest is that WM has been linked to a number of important skills, such as academic achievement and general intellectual capacity (Engle, 2002; Shipstead, Redick, \& Engle, 2010). Moreover, WM deficits often occur in common clinical conditions, such as dyslexia, ADHD, and major depression, as well as in normal aging (Lezak, Howieson, \& Loring, 2004). As a system for shortterm maintenance and manipulation of task-relevant information (Baddeley, 2000), WM is inherently involved in all higher-level cognitive activities. Accordingly, WM training, if successful, might have wide-reaching consequences for an individual.

The results from the initial WM training studies were very promising, because they suggested that it is possible to improve performance not only on the trained task but also on untrained tasks measuring other cognitive functions (Jaeggi, Buschkuehl, Jonides, \& Perrig, 2008; Klingberg, Forssberg, $\&$ Westerberg, 2002). The initial enthusiasm, however, turned into a controversy as subsequent training studies reported mixed results (Brehmer, Westerberg, Bäckman, 2012; Bürki, Ludwig, Chicherio, \& De Ribaupierre, 2014; Bäckman et al., 2011; Chooi \& Thompson, 2012; Colom et al., 2013; Dahlin, Neely, Larsson, Bäckman, \& Nyberg, 2008; Jaeggi et al., 
2008, 2010; Klingberg et al., 2002, 2005; Kundu, Sutterer, Emrich, \& Postle, 2013; Lilienthal, Tamez, Shelton, Myerson, \& Hale, 2013; Oelhafen et al., 2013; Redick et al., 2013; Salminen, Strobach, \& Schubert, 2012; Thompson et al., 2013; Waris, Soveri, \& Laine, 2015). The key issue is the existence of generalization following cognitive training, because the goal is to elicit positive transfer effects on untrained tasks. Most training studies make a distinction between near and far transfer effects. Near transfer refers to enhanced performance in a task that is intended to measure the trained cognitive domain and far transfer to improvement in another cognitive domain, such as WM training leading to better performance in a task measuring intelligence (von Bastian \& Oberauer, 2014). Because WM training studies have reported near transfer, far transfer, both near and far transfer, or no transfer at all, it has been difficult to draw conclusions about the efficacy of WM training. What complicates the matter further is that many of the previous training studies have suffered from methodological shortcomings, such as using small sample sizes, employing a no-contact control group, failing to randomly assign participants to groups, or using only a single task to measure a given cognitive ability (Melby-Lervåg \& Hulme, 2013; Melby-Lervåg, Redick, \& Hulme, 2016; Morrison \& Chein, 2011; Shipstead, Redick et al., 2010; Shipstead, Redick, \& Engle, 2012).

\section{Previous meta-analyses on working memory training}

Given the widespread interest in WM training and the large variability in results, it is not surprising that during the past five years a number of meta-analyses have addressed the outcomes of WM training (Au et al., 2015; Dougherty, Hamovitz, \& Tidwell, 2016; Melby-Lervåg, \& Hulme, 2013; MelbyLervåg \& Hulme, 2016; Melby-Lervåg et al., 2016; Schwaighofer, Fischer, \& Bühner, 2015; Weicker, Villringer, \& Thöne-Otto, 2016; for meta-analyses investigating not only WM training, see Hindin \& Zelinski, 2012; Karbach, \& Verhaeghen, 2014; Karr, Areshenkoff, Rast, \& GarciaBarrera, 2014; Kelly et al., 2014). Near and far transfer effects of different kinds of WM training have been studied in four meta-analyses (Melby-Lervåg \& Hulme, 2013; Melby-Lervåg et al., 2016; Schwaighofer et al., 2015; Weicker et al., 2016). The results from these meta-analyses (Table 1) with both clinical and healthy samples of children and adults showed that WM training can produce small to large short-term near transfer effects (verbal and visuospatial WM tasks) and null to small effects of transfer to verbal and visuospatial reasoning (or fluid intelligence; Gf) tasks. The results also showed small transfer effects to cognitive control (Melby-Lervåg \& Hulme, 2013; Weicker et al., 2016) and attention (Weicker et al., 2016), but no transfer to long-term memory (Weicker et al., 2016), arithmetic skills (Melby-Lervåg \& Hulme, 2013;

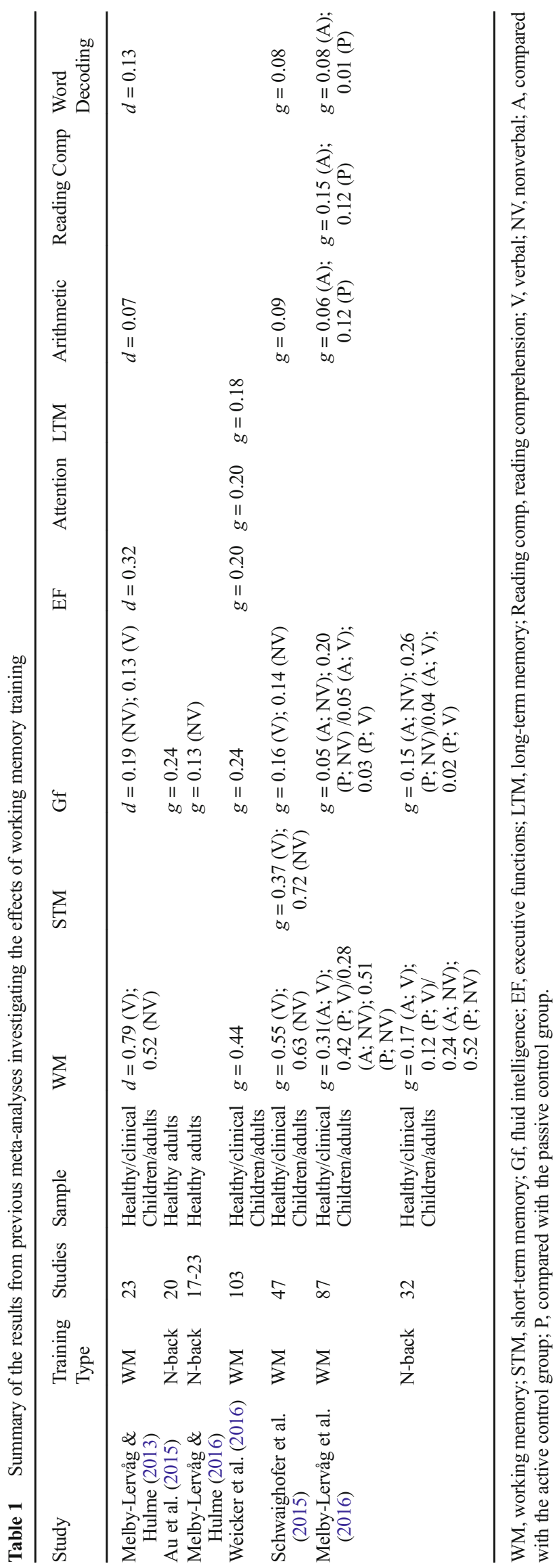


Schwaighofer et al., 2015; Melby-Lervåg et al., 2016), reading comprehension (Melby-Lervåg et al., 2016), or word decoding (Melby-Lervåg \& Hulme, 2013; Schwaighofer et al., 2015; Melby-Lervåg et al., 2016).

As is evident from the short review above, there is some disagreement between the previous meta-analyses, particularly regarding the size of the near transfer effects. These discrepancies are at least partly due to differences in decisions regarding the studies included in the meta-analyses and the categorization of tasks into different cognitive domains. For example, the meta-analysis by Melby-Lervåg et al. (2016) included studies that were not randomized, controlled trials. Furthermore, all previous meta-analyses included only one measure per domain per study. In some cases, this measure was a single task selected to represent a domain in a study, whereas in others, a mean value across the tasks was used. Moreover, the choice of which tasks to include varies between the meta-analyses. For example, unlike the other meta-analyses, Schwaighofer et al. (2015) did not include the n-back task in the near transfer measures due to validity issues with this task. Also, simple span tasks, such as the digit span, were considered WM measures in the Melby-Lervåg and Hulme (2013) study, whereas the other meta-analyses coded simple spans as measures of short-term memory (STM; Schwaighofer et al., 2015; Weicker et al., 2016) or excluded them completely from the analyses (Melby-Lervåg et al., 2016). Finally, a close look at the measures in Melby-Lervåg and Hulme (2013) showed three instances where the trained task itself was included in the near transfer analyses, likely leading to an undue inflation of the corresponding effect size. Of the previous meta-analyses, Melby-Lervåg and Hulme (2013) reported the strongest effect sizes for near transfer. Given these issues concerning the previous meta-analyses, further meta-analyses in this popular research field are warranted.

\section{Background for the present study}

The variety of WM training programs, experimental designs, and participant groups makes it challenging to draw definite conclusions on the WM training outcomes. To limit three sources of variability (training program, age, health status), the present meta-analysis focused solely on studies with healthy adults who trained with the most commonly used computerized experimental WM training paradigm, namely with n-back tasks. In the n-back task (Kirchner, 1958), the participant is presented with a sequence of stimuli and the task is to decide for each stimulus whether it is the same as the one presented $n$ trials earlier. In a single $\mathrm{n}$-back task, the participant is required to attend to one stream of stimuli, and in a dual n-back task, two streams of stimuli are presented simultaneously. Figure 1 shows an example of a dual n-back task with an auditory-verbal and a visuospatial stream of stimuli.

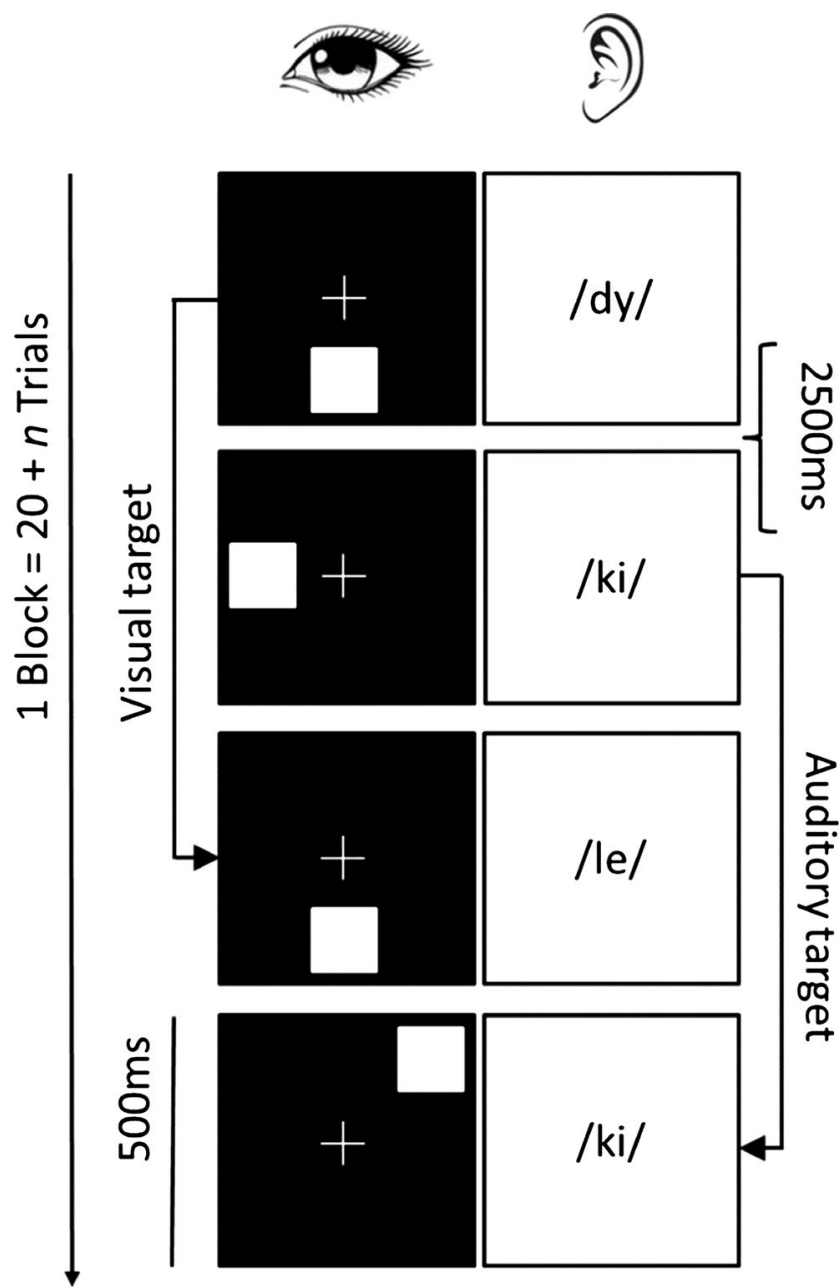

Fig. 1 Depiction of the dual n-back task. In a 2-back version, the participants should press the corresponding "yes" button for the third location and for the fourth syllable.

Previous studies comparing the effects of single and dual nback training have shown that both task versions seem to be equally effective in producing generalization and that the transfer effects are fairly similar (Jaeggi et al., 2010; Jaeggi, Buschkuehl, Shah, \& Jonides, 2014). The n-back task often is highlighted as a WM updating measure (Szmalec, Verbruggen, Vandierendonck, \& Kemps, 2011), but it also reflects active maintenance and capacity of the WM (plus interference control if so-called lure stimuli are included in the stimulus sequence; see NIMH Research Domain Criteria Workshop on Working Memory, 2010). The concurrent validity of the n-back task, however, has been questioned as previous studies have reported low correlations between the n-back task and other WM tasks, especially complex span tasks (Miller, Price, Okun, Montijo, \& Bowers, 2009; see Redick \& Lindsey, 2013, for a meta-analysis). Nevertheless, a recent study showed that at a latent level, the n-back task is highly correlated with other WM tasks (complex spans, WM updating tasks, and sorting spans; Schmiedek, Lövdén, \& Lindenberger, 2014). 
The efficacy of n-back training has previously been investigated in three recent meta-analyses (Au et al., 2015; MelbyLervåg \& Hulme, 2016; Melby-Lervåg et al., 2016). Au et al. (2015) focused solely on the training effects on Gf. They included 20 studies with healthy adults in their analyses and found a small transfer effect of $n$-back training to Gf ( $g=$ 0.24). Au et al. (2015) also investigated the effects of various moderators, such as the type of control group (active or passive), type of n-back task (single or dual), and type of material in the Gf transfer tasks (matrix or nonmatrix; verbal or visuospatial). Active control groups typically receive the same amount of training as the training groups do, but with tasks that are not intended to tap WM to any greater extent. Passive control groups only participate in pre- and postsessions. The results from the $\mathrm{Au}$ et al. (2015) meta-analysis indicated that studies with passive control groups showed more transfer to Gf than studies with active control groups. However, their followup analyses revealed that this finding did not stem from a difference between active and passive control groups but from the training groups for some reason performing better in studies with passive controls than in studies with active controls. According to Au et al. (2015), these results do not support the idea that the Hawthorne or expectancy effects affect the results. Finally, their results showed no effects of the other aforementioned moderators. The Au et al. (2015) meta-analysis was challenged by Melby-Lervåg and Hulme (2016) who criticized the exclusion of relevant studies, the calculation of effect sizes without taking pretest differences between groups into account, and the interpretation of the results comparing active and passive control groups. Melby-Lervåg and Hulme (2016) argued that even though the difference between active and passive control groups was not statistically significant in the Au et al. (2015) meta-analysis, there was a difference in the pre-post effect sizes between those two groups. They also emphasized that the analysis only consisted of 12 studies in each category. Melby-Lervåg and Hulme (2016) reanalyzed the Au et al. (2015) data by including only the nonverbal reasoning tasks and correcting for possible pretest differences between groups. They found a very small but statistically significant effect of transfer to these tasks $(g=0.13)$. Au et al. (2016) have later responded to this critique, and they maintain that the discrepancy between the effect sizes in the Au et al. (2015) and Melby-Lervåg and Hulme (2016) analyses is mainly related to differences in the various meta-analytic decisions, such as the fact that Melby-Lervåg and Hulme (2016) compared the same treatment group to several control groups without taking the dependency between these comparisons into account. Furthermore, to avoid confounds stemming from methodological differences between studies, $\mathrm{Au}$ et al. (2016) compared active control groups to passive control groups within such n-back training studies that had employed both. The results from those analyses showed no difference between the two types of control groups.
In their recent meta-analysis on different kinds of WM training, Melby-Lervåg et al. (2016) included the type of training paradigm as a moderator in their analyses. These results showed null to moderate training effects from $n$-back training to untrained WM tasks (verbal WM: $g=0.17$ for studies with active controls and $g=0.12$ for passive controls; visuospatial WM: $g=0.24$ with active controls and $g=0.52$ with passive controls) and very small but statistically significant transfer effects to nonverbal problem solving ( $g=0.15$ with active controls and $g=0.26$ with passive controls). Melby-Lervåg et al. (2016) further pointed out that those n-back training studies with active control groups that showed the largest transfer effects to nonverbal ability all had small sample sizes (less than 20 participants per group) and most of them also showed decreases in performance from pretest to posttest in the control groups. Melby-Lervåg et al. (2016) emphasized that only studies with active control groups can be used to evaluate the effectiveness of an intervention and they "recommend that investigators stop conducting working memory training studies with untreated control groups and that journals stop publishing them" (p. 524).

Compared with the earlier meta-analyses on transfer following n-back training, the present one has several distinctive features that we deem as important. First, as mentioned, limiting the sources of variability (here training method, age, and health status) should yield results that are easier to interpret. Second, we strictly separated the trained tasks from the untrained ones as mixing these would risk inflating the near transfer effect (see above for the discussion on the meta-analysis by Melby-Lervåg \& Hulme, 2013). Third, meta-analytic near transfer measures have lumped together untrained WM tasks that differ from the training tasks only by their stimuli and WM tasks that are structurally different from the training tasks (Melby-Lervåg \& Hulme, 2013; Melby-Lervåg et al., 2016; Schwaighofer et al., 2015; Weicker et al., 2016). We believe that one should keep these two transfer measures apart to obtain a more detailed view on the generalizability of WM training. For example, in nback training studies a near transfer effect rising only from untrained n-back tasks could speak for acquisition of taskspecific strategies rather than improved WM per se. Fourth, all previous meta-analyses have included only one measure (one task or average of several tasks) per domain per original sample in the analyses. The rationale for choosing a specific task to represent a certain domain is not always fully explicated. For example, in the meta-analysis by MelbyLervåg and Hulme (2013), there seems to be some inconsistency in the selection of tasks so that a specific task is excluded for some studies but not for all. In the present meta-analysis, we were able to include all measures from the original studies by employing a multilevel metaanalytical approach, and thereby having less risk of biasing the estimated training effect. 


\section{Structure of the present meta-analysis}

In the present meta-analysis on WM training with n-back tasks, we investigated the transfer to untrained tasks representing the following four cognitive domains: taskspecific near transfer as measured by untrained versions of the n-back task, WM as represented by other than n-back tasks, cognitive control, and Gf. Cognitive control was included as a transfer domain because earlier studies suggest that WM is closely related to other executive functions (Miyake et al., 2000). Transfer to attention and cognitive control has also been studied in two of the previous meta-analyses. Melby-Lervåg and Hulme (2013) included only the Stroop task in their analyses and Weicker et al. (2016) investigated two executive domains: cognitive control and executive functioning, and attention and processing speed. Both of these domains included tasks that can be considered as measures of inhibition (e.g., the Stroop task in the former and the Simon and Flanker tasks in the latter one). In our cognitive control domain, we included tasks that measure inhibition of irrelevant information, set shifting, multitasking, and verbal executive functions (e.g., verbal fluency). We excluded tasks that measure attention (e.g., 0-back). Finally, in line with previous meta-analyses we included fluid intelligence (verbal and nonverbal reasoning) as a separate domain in the analyses. These four domains also form a rough dimension of the degree of cognitive overlap between the training tasks and the transfer tasks. If the magnitude of transfer effects follows the presumed cognitive overlap (Dahlin et al., 2008; Waris et al., 2015), a decreasing degree of transfer should be seen when moving from n-back transfer tasks to WM, cognitive control, and Gf. However, as some previous studies suggest that the correlations between n-back tasks and other WM tasks are very low (Redick \& Lindsey, 2013), it is possible that the transfer effects to untrained WM tasks are lower in this meta-analysis than in those previous meta-analyses that investigated various kinds of WM training. This also appears to be the case in the analyses on n-back training in the MelbyLervåg et al. (2016) meta-analysis.

Several moderating factors were taken into account in the present meta-analysis, namely the type of control group (active vs. passive), type of training (single vs. dual n-back), training dose (small vs. large), transfer materials (verbal vs. nonverbal), and age (young vs. old). Following the current discussion on the effects of the type of control group ( $\mathrm{Au}$ et al., 2016), we compared active and passive controls only within studies (comparing active and passive control groups within those studies that have employed both). We also used the type of control group as a covariate in our analysis of the training effect, thereby considering the potential influence of the type of control group used in an experiment.

In addition, we implemented some methodological improvements that allowed us to more fully take advantage of the information in the original studies. Because the training of participants is quite resource intensive, a study in this field typically contains a relatively small sample measured on several dependent measures, both before and after training. For a meta-analysis, the information value of a single original study with a small sample is quite limited (for a discussion on this problem in the context of working memory training, see Bogg \& Lasecki, 2014), but it can be enhanced by adequate modeling allowing for inclusion of several measures and by utilizing the advantages of the repeated measures design.

Therefore, we made use of a multilevel meta-analytical approach to include effect sizes for all pertinent tasks from the original studies while accounting for the fact that these effect sizes came from the same sample. The multi-level approach has two general advantages. First, it allows us to include more effect sizes and thereby gives us a better picture of the differences in the general training effects between studies. Second, it allows for a more complete comparison of improvements on different tasks within the same study, providing data for the aforementioned moderation analyses. Furthermore, we took full advantage of the repeated measures design used in the original studies. A repeated measures design offers higher statistical power than an independent groups design, but only if one accounts for how strongly the repeated measures are correlated. If this is disregarded, as is the case in the previous metaanalyses in this field, the standard errors of the effect sizes are inflated to that of independent group designs. The effect sizes themselves are not affected, but differences in reliability (for other reasons than sample size) can only be caught by comparing each individual's posttest measure with the corresponding pretest measure. To do this, one needs to analyze the original data or, as in our case, account for the correlation between preand posttest measures. Besides more accurately allocating the appropriate relative weight to each study, a more crucial advantage is seen in the increased statistical power. Thus, by accounting for the correlation between pre- and posttest measures, we raise the statistical power of our meta-analysis, which allows us to make use of the multi-level approach and more confidently examine moderation effects.

Our research questions were as follows:

1. Does n-back training improve performance on other untrained n-back tasks, structurally different WM tasks, cognitive control tasks, or fluid intelligence tasks?

2. Is the type of control group (active vs. passive) related to the transfer effects in the four domains?

3. Is the type of n-back training task (single vs. dual) related to the transfer effects in the four domains?

4. Is the effect of dual n-back training different for verbal and nonverbal transfer tasks?

5. Is age related to transfer effects in the four domains?

6. Is the duration of training related to transfer effects in the four domains? 


\section{Method}

\section{Literature search and criteria for inclusion}

We searched the electronic databases PubMed, PsycINFO (ProQuest), Google Scholar, Google, and ERIC for relevant research reports (articles, dissertations, and theses). The first search was conducted in February 2015. The search term $n$ back training was used in all databases. In Google Scholar and Google, citation marks (" $n$-back training") were used to reduce noise in the search. The first search resulted in 99 hits in PubMed, 86 in PsycINFO, 189 in Google Scholar, 12,500 in Google, and 5 in ERIC. We screened all hits in the databases with the exception of Google, where we limited screening to include only the first 100 hits. For a study to be included at this stage, the following criteria had to be met:

1. A randomized, controlled trial with at least one training group and at least one control group and a pretest-posttest design.

2. Transfer tests were employed within any of the following cognitive domains: n-back, WM, fluid intelligence, and cognitive control.

3. Training program included only n-back task(s).

4. Training task in the active control group was not a WM task.

5. Sample consisted of healthy adults.

Search hits were screened in the aforementioned order of the databases. Our inclusion criteria yielded 12 studies from PubMed, 8 additional studies from PsycINFO, 12 additional studies from Google Scholar, and 2 additional studies from Google (all numbers refer to unique studies; the number of overlapping studies is not reported). Our search thus resulted in 34 reports. In the next step, three theses were excluded because the data also were included in published articles, and one thesis was excluded because different transfer measures were used before and after training. Two theses had been written on the same data collection and therefore were treated as one study. The initial literature search thus resulted in 29 studies.

The literature search was updated three times: once in July 2015, once in February 2016, and once in October 2016. The search procedure was the same as in the initial literature search. These searches resulted in the inclusion of six additional studies: one from the first search, one from the second search, and four from the last search. The reference lists of the included studies and prior meta-analyses in the field were also screened, and as a result, six potentially interesting studies were found. Moreover, a yet unpublished study conducted by two of the authors (Soveri, Karlsson, Waris, Grönholm-Nyman, \& Laine, submitted) of the present meta-analysis was included.

In sum, the total number of studies identified for inclusion was 42 . The authors of 18 of these studies were contacted to receive necessary information not reported in the published articles. We received the requested data for nine of these. However, the remaining nine articles had to be excluded, because we did not receive the information or the authors informed us that the requested data were reported in other articles already included in our sample of studies. Therefore, our final sample consisted of 33 studies. Figure 2 depicts a flow chart of the screening process.

We have chosen to exclude some of the n-back training studies with healthy adults that have been included in previous meta-analyses. These studies are listed in Table S1 together with the reason for exclusion. Furthermore, the Seidler et al. (2010) study in Au et al. (2015) is referred to as Anguera et al. (2012) in the present meta-analysis.

\section{Data coding}

Type of group The type of group was described with a variable with three levels: "Training group"; "Active control group"; and "Passive control group." The criterion for consid ering a control group as an active control group was that participants received the same amount of training as the training group, but that, purportedly, their training program did not load on WM. Passive control group participants did not receive any intervention and participated in the pre- and posttests only.

Training task The training tasks were described by two variables. "Training type" described whether a single or dual (or, in one case, a triple) n-back task was employed. In a single nback task, the participants responded only to one stream of stimuli, while the dual n-back task consisted of two streams that were presented simultaneously. "Training materials" defined whether the training task had been verbal, nonverbal or both verbal and nonverbal. The n-back task was considered verbal when the stimuli consisted of language materials such as numbers, letters, or syllables. Task versions without verbal elements were coded as nonverbal. Those training tasks that included both verbal and nonverbal stimuli were exclusively dual n-back tasks.

Training dose Training dose was operationalized with two variables: "Training hours" and "Training sessions." The former one corresponded to the total number of hours the participants had been training and the latter one to the total number of training sessions. Due to an uneven distribution, we dichotomized the two variables based on a median-split. For the amount of training, the levels were: 6.67 hours or less and more than 6.67 hours. When the information about training dose was imprecise (e.g., 20-25 minutes per session), the lowest number was used to calculate the total amount of training. 


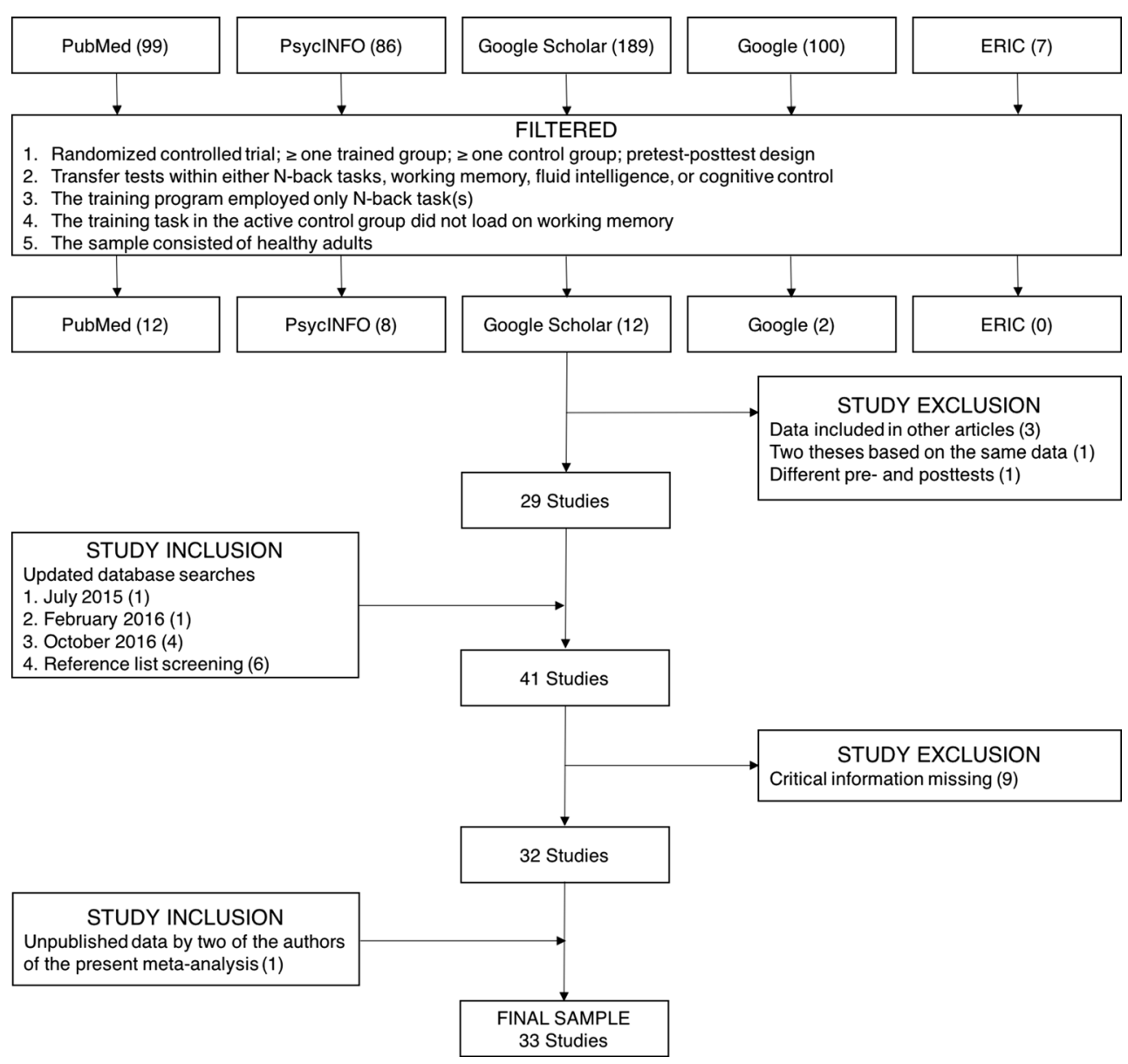

Fig. 2 Search for literature and the screening process.

The median-split for training sessions was as follows: 15 sessions or less, and more than 15 sessions.

Transfer tasks Transfer was described by two variables: "Transfer domain" and "Transfer materials." "Transfer domain" assigned transfer tasks to one of the following cognitive domains: n-back, WM, cognitive control, and Gf. All untrained versions of the n-back task were coded as n-back transfer measures, that is, measures of task-specific transfer. The remaining WM tasks employing some other paradigm than $\mathrm{n}$ back were coded as WM transfer tasks. We included, for example, simple span tasks (e.g., digit span), complex span tasks (e.g., operations span), and running memory span tasks (e.g., letter-memory). In our cognitive control domain, we included tasks that tap inhibition of irrelevant information (e.g., Stroop and Simon task), set shifting (e.g., Number-letter task), multitasking (e.g., SynWin), and verbal executive functions (e.g., verbal fluency). Tasks that can be considered as measures of attention and vigilance were not included in the cognitive control domain. Finally, all tasks where the participant was required to solve problems without being able to rely on previous experience to any greater extent, were coded as measures of Gf. Reading comprehension was not included in the
Gf measure due to the fact that in those tasks, the correct answer is available in the testing materials. The few transfer tasks that did not fit into these categories were excluded from the analyses due to the low number of tasks per remaining domain. One of these was the 0-back condition of the nback task, which was not included as an n-back measure, because it exerts only a minimal load on WM. Neither was it coded as a measure of cognitive control, because it is a vigilance task and a variant of the trained task.

For some tasks, several measures were reported. For those tasks for which both reaction times (RT) and accuracy were available, we decided to code only the RT measures as on some tasks accuracy rates are plagued by ceiling effects. Also, for such tasks that included several conditions (e.g., single task trials, repetition trials, and switching trials in set shifting tasks), we decided to include difference scores (e.g., the switching cost, which is the difference between switching trials and repetition trials) when possible. In case these were not available, we included the conditions that involved executive load (i.e., the switching condition).

In order to investigate whether transfer was more likely to the verbal or nonverbal domain, the variable "Transfer materials" was created. Here, the two transfer domains "Gf" and 
"WM," for which we obtained the largest number of comparisons, were further divided into verbal versus nonverbal reasoning, and verbal versus nonverbal WM.

The coding of transfer tasks into cognitive domains (i.e., task-specific transfer as measured by n-back, WM, cognitive control, and Gf), and materials (i.e., verbal vs. nonverbal), was based on consensus decisions by three of the authors (A.S., M.L., and L.K.).

Age Age was dichotomized due to data-availability issues (mean ages were reported in some studies and age ranges in others) and because of its non-normal distribution. The variable "Age group" was coded in the following manner: 59 years or younger, and 60 years or older. This division was made based on the age ranges reported in the studies. For studies that did not include information about age range, the decision was made based on other kinds of information provided in the studies (e.g., university students and/or the mean age and its standard deviation for the sample).

Furthermore, we coded the publication year of the articles and theses and the countries where the studies were conducted. More detailed information about the abovementioned variables in each original study is given in Tables S2 and S3.

Statistics for effect size calculation Group means, standard deviations, and group sizes were extracted for each group to calculate a measure of gain score for each group per transfer measure. To obtain the correlations between pre- and posttest performance (such correlations were reported for all measures in only one of the studies), we contacted the authors of the original studies. After the authors had responded, we had correlations for 406 of the 507 within-sample pre-post comparisons. Where authors did not provide the correlations, we attempted to deduce the correlation from other available information in the study (e.g., the means and standard deviations in combination with a $t$ or $F$-value). We could deduce or give a conservative estimate for 80 of the 101 remaining pre-post correlations. Where we were unable to estimate the correlation, we entered 0 as a correlation, essentially treating the pre- and posttest measures as independent groups. The effect of using a conservative correlation is that the within-sample pre-post comparison is given a larger standard error and therefore less weight in the meta-analysis. It is appropriate that we give less weight to studies where we are less certain about the information provided in the summation of effect sizes, but overly conservative estimates for the standard errors cannot be included in analyses where the standard errors are of primary interest, such as a publication bias analysis. A meta-analysis of the prepost correlations showed that the correlations were $r=0.659$ ( $k$ $=203, p<0.001)$ for the pre-post measures in the training group, $r=0.649(k=94, p<0.001)$ for the pre-post measures in the active control group, and $r=0.656(k=109, p<0.001)$ for the pre-post measures in the passive control groups.
To address the reliability of our coding of group sizes, means, and standard deviations, we conducted a test of interrater reliability. Ten of the 33 studies were randomly selected and recoded by an independent researcher and the percentage of agreement (i.e., same value or not) was calculated. The interrater agreement was $97.1 \%$ for the 34 group size values, $100.0 \%$ for the 284 mean vales, and $99.6 \%$ for the 284 standard deviation values in the ten studies. Closer scrutiny revealed two points of disagreement. When we returned to the studies to retrieve the correct information, we found that the first disagreement was an error made by the independent researcher. Because the second disagreement amounted to less than one decimal point, we deemed the coding as reliable.

\section{Meta-analytical procedures}

All effect sizes were calculated and all meta-analyses were conducted using the Metafor package (Viechtbauer, 2010) for R (R Core Team, 2008).

Calculation of effect sizes The effect sizes of interest were pairwise comparisons of pre-post changes (i.e., the difference in performance between the first and second cognitive test sessions) between a training group and a control group. To obtain these effect sizes, we first retrieved the within-group pre-post effect sizes $\left(g_{\text {WITHIN }}\right)$ for each group and measure included in a study. For $g_{\text {WITHIN }}$, we calculated the standardized mean pre-post change for each training and control group separately using the escalc function in the metafor package (Viechtbauer, 2010). In the function documentation, this effect size is called a "standardized mean change using raw score standardization" (SMCR). Here, the raw gain score on a task is standardized by dividing it by the standard deviation of the pretest score, and the variance of the effect size is adjusted by accounting for the correlation between the pre- and posttest measure. To make the $g_{\text {WITHIN }}$ effect sizes comparable between training and control groups, we first pooled the standard deviation across all groups within the same experiment.

To calculate an effect size representing the training effect $\left(g_{\text {BETWEEN }}\right)$, that is, a between-groups effect size representing the difference in pre-post change between a training group and a control group, we subtracted the $g_{\text {WITHIN }}$ for the control group from the $g_{\text {WITHIN }}$ for the training group. A positive value thus indicates that the pre-post improvement was larger (or, that the pretest-posttest decline was smaller) in the training group compared to the control group. The variance for $\left(g_{\text {BETWEEN }}\right)$ was calculated by summing the variance of the within-group effect size for the two groups.

Multi-level modeling Six of the 33 studies (i.e., articles, theses, or manuscripts) contained more than one experiment. Here, separate experiments have their own set of training 
and control groups. For example, one experiment might compare a training group of young individuals to a control group of the same age, while another experiment compares training and control groups with older individuals. Because one of the studies contained four experiments and four studies contained two experiments, a total of 41 experiments were included from the 33 studies. Experiments also varied in the number of training and/or control groups included. Twelve experiments (in 11 studies) included more than one training group. Eight other experiments (in six studies) involved both an active and a passive control group. The 41 experiments included, in total, 103 different training or control groups. These groups had been measured before and after training, for between one and 13 different measures, depending on the study and experiment. In total, the meta-analysis contained 507 within-sample prepost comparisons $\left(g_{\text {WITHIN }}\right)$ from a total of 2105 participants.

These 507 within-group effect sizes yielded 305 betweengroups effect sizes (i.e., comparisons between a training group and a control group within an experiment). There were, however, several forms of dependency between effect sizes in this structure. Effect sizes that are dependent are generally less informative than independent ones. When two measurements are perfectly correlated (e.g., measuring the improvement in WM with two or more entirely interchangeable measures), these measurements will provide the same information. This means that including interchangeable measurements, while assuming that they provide independent and unique information, unduly inflates the confidence in the results of a metaanalysis. In other words, unwarranted repetition of the same information will lead to an incorrect narrowing of the confidence estimates in a meta-analysis, which, in turn, increases the risk of making type I errors (Becker, 2000; van den Noortgate, López-López, Marín-Martínez, \& Sánchez-Meca, 2013).

The following forms of dependency between effect sizes need to be considered: The most obvious dependency is that when multiple tasks are used to measure the training effect within the same experiment, the outcomes on these tasks are likely correlated. In other words, these outcomes represent the training effect within the same participant(s). Second, effect sizes for a training effect are also correlated when a particular training group is compared with multiple control groups within an experiment. Third, and similarly, effect sizes are correlated when multiple training groups are compared with one control group. Fourth, and lastly, effect sizes from multiple experiments within a particular study may be correlated due to similarities in experimental methodology and the experimental setting.

With an adequate amount of data, the correlation within each of these levels of dependency can be estimated in a multilevel framework. This model is, however, too complex for the amount of data available, but a few simplifications can be made that will make modelling considerably more parsimonious with little loss of information and with few added assumptions: (a) The experiments in the four studies that had more than one experiment were too few to allow us to estimate the variance between experiments within studies. All experiments, however, have separate participants and the dependency within participants is therefore handled adequately. Thus, for the sake of parsimony we did not model the dependency between experiments within the same study, but treated all 41 experiments as independent samples; (b) When there were more than one training group, we performed an internal fixed effects meta-analysis of the $g_{\text {WITHIN }}$ in the groups producing a single $g_{\text {WITHIN }}$ for the training groups in that experiment, and in the experiments where both an active and a passive control group was used we disregarded the passive group (except for the comparisons between control groups within studies). These simplifications limit each experiment to one single comparison between a training group and a control group per measure. This eliminates dependency between comparisons within an experiment that would reuse training or control groups.

What remained were 203 estimated training effects (i.e., comparisons of training vs. control group) in 41 separate experiments. These comparisons, in this framework, are presented in Fig. 3 (see Table S3 for more detailed information about pre-post effect sizes and group comparisons). Hence, our model is a three-level model with random intercepts for each unique experiment and for each unique measure within those experiments. In other words, in addition to observing the variance of participants' results on a given measure in a given experiment, we estimate the variance of the mean results of different experiments, and the variance of mean results for different measures within an experiment. Thereby, we can account for, and estimate, the dependency of effect sizes between-measures within experiments.

This dependency, or how strongly measurements are clustered within experiments, can be estimated through the intraclass correlation coefficient (ICC). It can be estimated by dividing the variance between experiments by the sum of the variance between and within experiments. The ICC, thus, also describes the amount of the variance in the effect sizes that can be attributed to differences between experiments. In Fig. 3, variance both between and within experiments can be discerned. When the variance within studies is small in relation to the variance between studies, the correlation within studies is deduced to be high. In contrast, if measures within studies vary to the point where each measure could just as well belong to any one of the experiments, the correlation is deduced to be close to zero. The $I C C$ for the clustering of training effect among measurements within an experiment was 0.499 . To evaluate whether the added model complexity paid off, we also conducted ANOVA tests. The test between the single- and two-level models was significant $(L R T=24.34, p$ $<0.001$ ), and so was the test between the two- and three level 


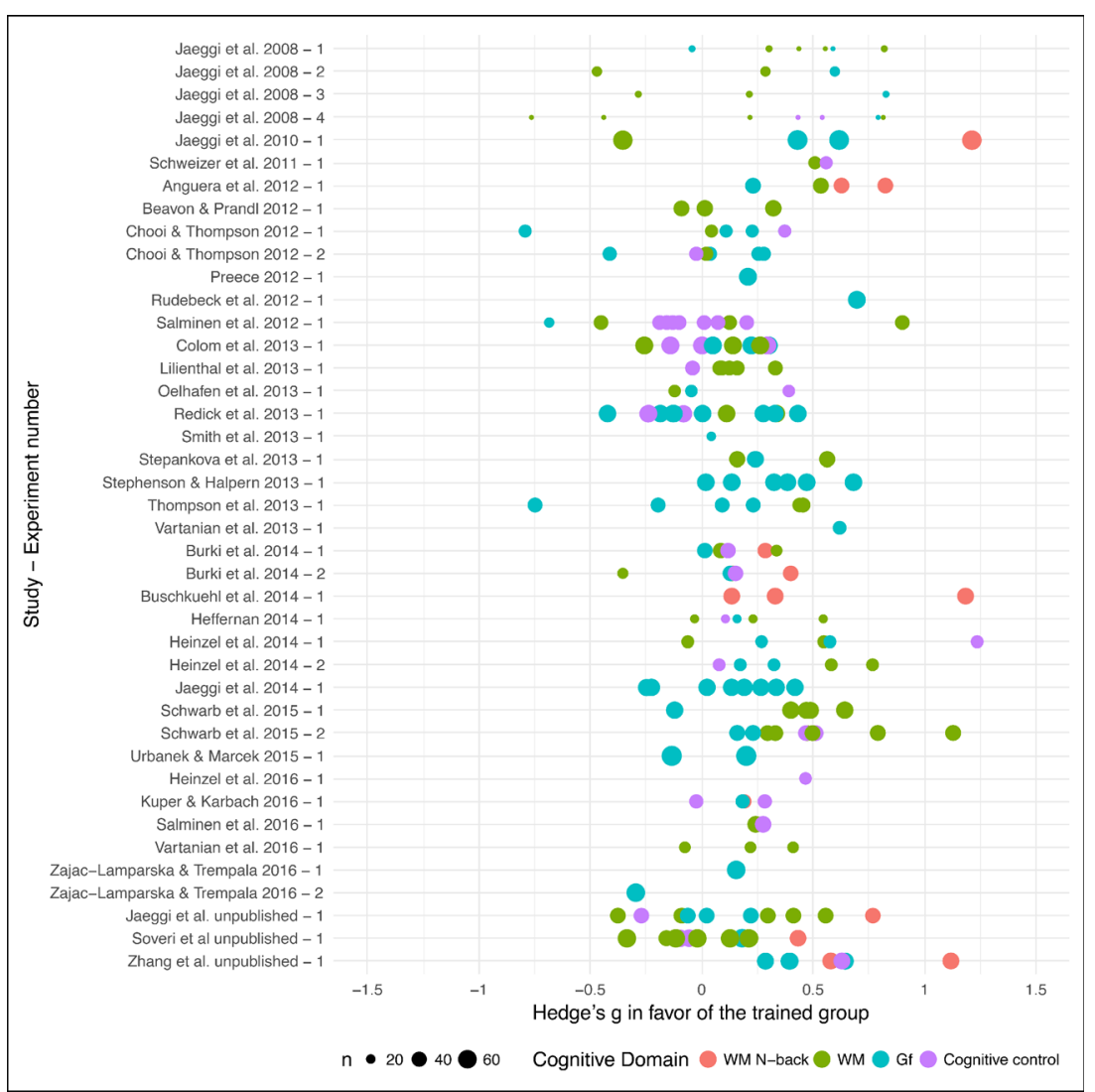

Fig. 3 Overview of the multi-variate and multiple-treatment structure of the included studies. The figure displays effect sizes, sample sizes, and cognitive domain (untrained n-back tasks [WM N-back], Working Memory [WM], Cognitive control, and Fluid Intelligence [Gf]). The horizontal axis indicates a Hedge's $g$, with positive effects (favoring training groups over control groups) to the right and vice versa. Two effect sizes are outside the plotted range.

that have not been reported in the final study). While selecting a subset of independent effect sizes could relieve the problem of dependency, this approach would lead to loss of information without resolving the problem of ghost variables. For our purposes, we find a careful visual inspection of funnel plots accompanied by regression-based methods to assess funnel plot asymmetry to be the most fruitful approach.

We find two forms of funnel plots especially informative for our purposes. One is the so-called contour enhanced funnel plot (Peters, Sutton, Jones, Abrams, \& Rushton, 2008) where each effect size is plotted against the precision (inverse of the standard error). The vertical reference line is drawn at 0 and the contours change shade at different levels of two-tailed $p$-values. When publication bias is absent, the individual effect size measures should be distributed symmetrically around the mean effect size, with measures with higher precision distributed more closely around the mean effect size. Visually, this typically forms an inverted funnel with the tip of the funnel further up in the plot and close to the mean effect size. Publication bias due to underreporting of non-significant findings should result 
in an asymmetrical funnel with effect size estimates missing in the area of the plot indicating nonsignificant results.

The second form of funnel plot that we find informative we call a funnel plot of residuals. Publication bias is only one possible explanation for funnel plot asymmetry. Another feasible explanation is systematic heterogeneity due to moderators (Egger et al. 1997). By plotting the residuals for the effect sizes in the model against the standard error (with lower standard error higher on the y-axis), the moderators in the model can be accounted for and the funnel plot asymmetry in relation to the expected value for each effect size measure can be examined. Similar contours as in the contour enhanced funnel plots can be added. In these plots, we included the betweenstudy and the between-measure variance in the total variance for an effect size. We used this plot to compare the effect sizes to the mean effect for each cognitive domain. Simultaneously, we accounted for the variance between and within studies. Thereby, we were able to use these plots to also examine outliers.

To more formally test for funnel plot asymmetry, we added the standard error or variance of the effect size as a predictor to pertinent meta-regression models. This is a close equivalent of the PET-PEESE method suggested by Stanley and Doucouliagos (2014), while simultaneously retaining our three-level model.

In the PET-PEESE approach, the effect size is first regressed on the standard error of the effect size in a weighted least squares (WLS) regression with the standard error of the effect size as the weight. Testing if the slope of the regression line is statistically significant serves as a test for statistically significant publication bias. The typical relationship when publication bias is present is that higher standard errors are associated with larger effect sizes. The intercept of this regression is interpreted as an estimate of the effect in a hypothetical study of no error $(\mathrm{SE}=0)$ and therefore no bias. Testing whether the intercept is statistically significant serves as a test of whether there is a true, bias - corrected, effect different from 0 . This test is called the precision-effect test (PET). In the simulation study by Stanley and Doucouliagos (2014), PET performed well when the true effect in the meta-analyzed studies was 0 , with the intercept being a slight overestimation of the true effect. When there was a true effect, however, using the variance as the predictor in the regression showed better performance. Here the intercept was a slight underestimation of the true effect. This test is called a precision-effect test with standard error (PEESE). The authors thus suggested that PET is followed up by PEESE if PET shows a true bias - corrected effect.

Stanley and Doucouliagos (2014) did not evaluate the method in a three-level model but we find this approach to be the best available method to estimate the true effect adjusted for possible publication bias. Furthermore, we find that adjusting for pertinent moderators in the same model is motivated to exclude the possibility that there are small-study effects associated with the moderators rather than explainable by publication bias. As such, this is a test most closely related to the funnel plot of the residuals.

We did not plot effect sizes where we could not estimate the pre-post correlation, as our conservative estimate (0) unduly inflates the standard error and those observations thus differ from the other combinations of effect sizes and their standard errors.

\section{Results}

\section{Descriptive results}

The study sample consisted of 33 studies, including 41 experiments, with, in total, 103 experiment groups. Of these groups, $54(52.4 \%)$ were training groups and $49(47.6 \%)$ were control groups. Twenty-three (42.6\%) of the training groups practiced with a single n-back task, 30 (55.6\%) with a dual n-back task, and $1(1.9 \%)$ with a triple n-back task. Twenty $(40.8 \%)$ of the control groups were active, and 29 (59.2\%) were passive. See Table 2 for a summary of study characteristics.

\section{Data screening and assessment of publication bias}

First, we investigated publication bias separately for each cognitive domain. We plotted the effect sizes in four separate contour-enhanced funnel plots: one for each domain. We then fitted a model with cognitive domain as a moderator and plotted the residuals in four separate funnel plots of residuals.

In the contour-enhanced funnel plots (Fig. 4, left column), each effect size is plotted against its precision $(1 / S E$, the inverse of the standard error). The vertical reference line is at $g=$ 0 . Contours change shade at different levels of two-tailed $p$ values. Publication bias due to underreporting of nonsignificant findings should result in an asymmetrical funnel with effect size estimates missing in the area of the plot indicating nonsignificant results. While there was some asymmetry, there was no obvious pattern of statistically significant effect sizes being preferred over nonsignificant effect sizes.

In the funnel plot of residuals (Fig. 4, right column), each effect size is plotted against the $S E$ with lower $S E$ higher up. The vertical reference line is at the aggregated effect size for the pertinent cognitive domain. The effect sizes are thus plotted in relation to the expected value on each domain respectively, with outliers falling outside the contour lines. Cognitive domain was indeed a statistically significant moderator, $Q_{M}$ $(3)=21.60, p<0.001$ (Fig. 4) 
Table 2 Number of experiments and number of training-control comparisons of measures per study characteristic

\begin{tabular}{|c|c|c|}
\hline Study characteristic & Number of experiments & Number of comparisons \\
\hline Total & 41 & 305 \\
\hline \multicolumn{3}{|l|}{ Training groups } \\
\hline Single n-back & 20 & 115 \\
\hline Dual n-back & 25 & 188 \\
\hline Triple n-back & 1 & 2 \\
\hline \multicolumn{3}{|l|}{ Control groups } \\
\hline Passive & 29 & 198 \\
\hline Active & 20 & 107 \\
\hline \multicolumn{3}{|l|}{ Cognitive domain } \\
\hline N-back & 9 & 17 \\
\hline Working memory ${ }^{\mathrm{a}}$ & 28 & 104 \\
\hline Verbal & 28 & 70 \\
\hline Nonverbal & 13 & 29 \\
\hline Fluid intelligence & 35 & 133 \\
\hline Verbal & 4 & 15 \\
\hline Nonverbal & 35 & 118 \\
\hline Cognitive control & 23 & 51 \\
\hline \multicolumn{3}{|l|}{ Sample age $(y r)^{b}$} \\
\hline Young & 33 & 271 \\
\hline Old & 5 & 27 \\
\hline \multicolumn{3}{|l|}{ Year } \\
\hline 2008 & 4 & 20 \\
\hline 2010 & 1 & 8 \\
\hline 2011 & 1 & 6 \\
\hline 2012 & 7 & 44 \\
\hline 2013 & 9 & 94 \\
\hline 2014 & 7 & 54 \\
\hline 2015 & 3 & 29 \\
\hline 2016 & 6 & 21 \\
\hline Unpublished & 3 & 29 \\
\hline \multicolumn{3}{|l|}{ Country of origin } \\
\hline Australia & 2 & 4 \\
\hline Canada & 3 & 9 \\
\hline Czech Republic & 2 & 12 \\
\hline Finland & 1 & 11 \\
\hline Germany & 6 & 33 \\
\hline Poland & 2 & 6 \\
\hline Spain & 1 & 9 \\
\hline Switzerland & 8 & 47 \\
\hline Taiwan & 1 & 8 \\
\hline United Kingdom & 2 & 8 \\
\hline United States & 13 & 158 \\
\hline
\end{tabular}

Young $=$ sample age younger than age 60 years; Old $=$ sample age 60 years or older

${ }^{a}$ For five of the comparisons in two experiments, the working memory task could not be categorized as verbal or nonverbal

${ }^{\mathrm{b}}$ Six samples in three experiments (7 comparisons) could not be categorized as either young or old
In these funnel plots, asymmetry is a little more apparent. For cognitive control and Gf, there is a group of effect sizes with high $S E$ that show higher effect sizes than the aggregated effect size for that domain. Furthermore, for all domains taken together, a group of effect sizes stands out, because their $S E$ is uncharacteristically high for this sample. An examination revealed 12 effect sizes with standard errors higher than 0.5 , which proved a natural cutoff point. Note that the standard error in the funnel plot is based on the combined variance from each level of the multilevel model and thus does not completely correspond to the standard error calculated for the effect size alone.

There are two reasons for excluding these 12 effect sizes from further analyses. First, removing them markedly reduces funnel plot asymmetry. Effect sizes with high standard errors are indeed most susceptible to publication bias, and removing them will only lead to a small decrease in statistical power. Second, these effect sizes are outliers in respect to their standard error and thereby they risk having a strong influence on PET-PEESE analyses, in which the standard error is used as a predictor.

The funnel plot of residuals also reveals two effect sizes for Gf that we considered outliers. These two had standardized residuals of 3.9 and -3.4 , respectively. One of these was also excluded based on its large standard error. We thus excluded 13 of the originally 203 effect sizes (see Table S3 for these exclusions).

\section{Training effects by cognitive domain}

We then investigated the transfer effects by cognitive domain. We found that the effect was largest for n-back, $g=0.62$, $[0.44,0.81], p<0.001$, followed by WM, $g=0.24$, [0.16, 0.32], $p<0.001$, cognitive control, $g=0.16$, [0.05, 0.27], $p$ $<0.001$, and Gf, $g=0.16$, [0.08, 0.24], $p<0.001$ (Fig. 5).

The moderation effect was significant $Q_{M}(3)=23.77, p<$ 0.001 . The effect on n-back tasks differed from effects on all other tasks (all $p \mathrm{~s}<0.001$ ). There were no significant differences between the effects for other domains (all $p \mathrm{~s} \geq 0.134$ ). The test for residual heterogeneity was also significant $Q_{E}$ $(186)=230.59, p<0.05$.

After this, we reran our main analysis with all data included. Again, the effect was largest for n-back, $g=0.63$, [0.44, $0.82], p<0.001$, followed by WM, $g=0.24$ [0.16, 0.33], $p<$ 0.001, cognitive control, $g=0.17,[0.06,0.29], p<0.001$, and Gf, $g=0.16,[0.08,0.24], p<0.001$. Note that although we find the exclusion of outliers and effect sizes with uncharacteristically high effect sizes beneficial to the precision of our analyses, the results are very similar before and after exclusion.

As a test for potential small-study effects that could remain even after our data screening, we added first the standard error and then the variance of the effect size as a predictor in PET- 

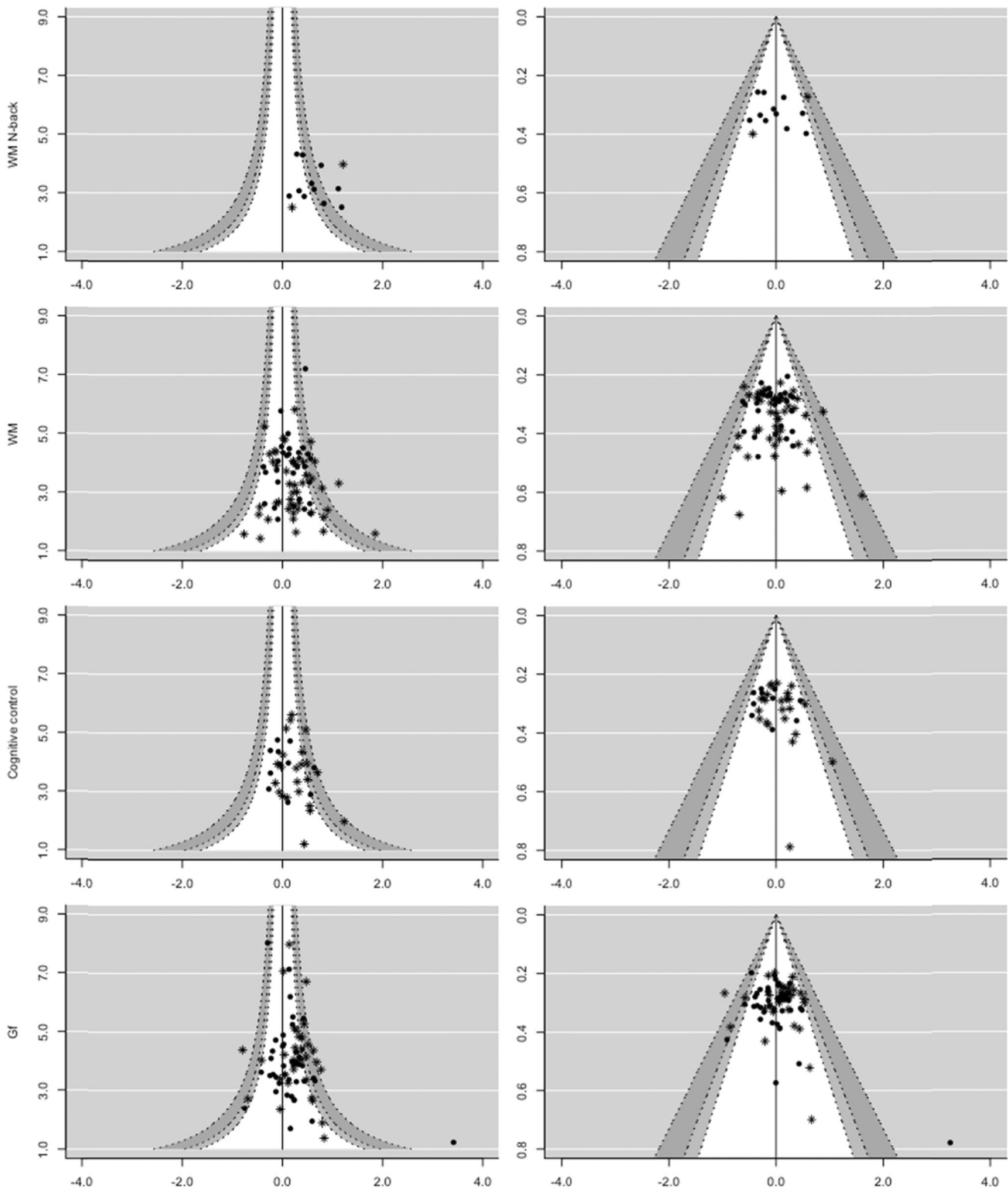

Fig. 4 Contour-enhanced funnel plots for each cognitive domain (by rows). In the funnel plots in the left column, effect sizes are plotted against their precision $(1 / S E)$. In the funnel plots in the right column,

effect sizes are plotted against the $S E$. The reference lines represent an effect size of Hedge's $g=0$ (left column) and the mean Hedge's $g$ within each domain (right column).

PEESE type analyses. Neither the standard error $(b=0.067$, $S E=0.338, p=0.844)$ nor the variance $(b=-0.037, S E=$

$0.599, p=0.951)$ were statistically significant predictors of effect size. Because the estimated effect sizes for the cognitive 


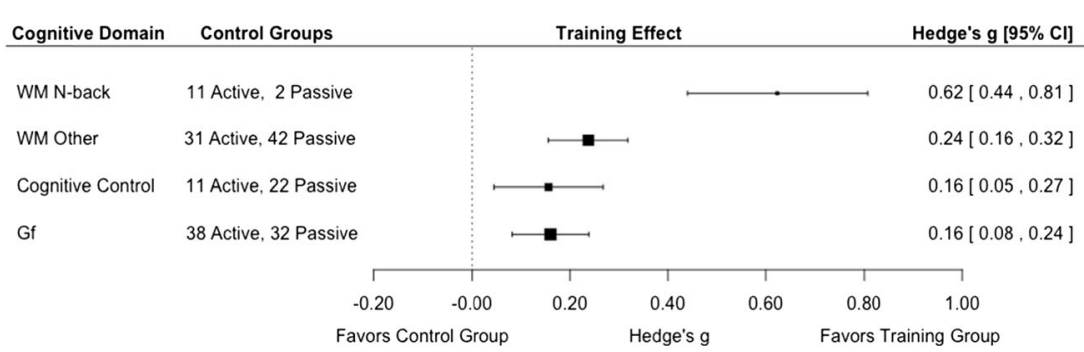

Fig. 5 For each cognitive domain, the figure displays synthesized effect sizes and confidence intervals for the comparison of pretes- posttest improvements between training groups and control groups. Positive values favor the training groups and negative values favor control groups.

domains were close to identical to the ones reported above in both analyses (the largest discrepancy was 0.02 points), we conclude that, after data screening, there was no evidence of publication bias affecting our conclusions.

\section{Control analyses}

The type of control group (active vs. passive), moderated slightly, but statistically significantly, the estimated training effect $(g=0.11,[0.00,0.22], p=0.048)$. After controlling for the type of control group, training effects were still largest for n-back, $g=0.59, p<0.001$, followed by WM, $g=0.18, p<$ 0.01 . The effect sizes for cognitive control was $g=0.08, p=$ 0.202 . For Gf the effect size was $g=0.11, p>0.05$. (Estimates for experiments with passive control groups are 0.11 points higher).

The analysis on transfer to WM was run also without simple spans so that the WM domain would be more similar to previous meta-analyses that have investigated simple spans separately. This resulted in a slightly smaller training effect for WM ( $g=0.21,[0.12,0.30], p<0.001)$, but the effect size was within the confidence intervals of the earlier estimate.

We also reran the analysis with n-back measures pooled with other measures of WM (including simple spans). This resulted in an increase of the training effect on WM, $g=$ 0.29 [0.21, 0.37], $p<0.001$.

\section{Efficacy of N-back training in different training contexts}

Furthermore, we investigated the effects of training type (single or dual n-back training), training dose (number of sessions and hours of training), and sample age (young or old) on the effects of N-back training. Training type (single or dual n-back training) did not produce significantly different training effects, $Q_{M}(1)=2.19, g=0.09[-0.03$, $0.21], p=0.139$, as there was a similar training effect for single n-back training, $g=0.27[0.18,0.37], p<0.001$, and dual n-back training, $g=0.18[0.11,0.26], p<0.001$. The test for residual heterogeneity remained significant, $Q_{E}(188)=254.86, p<0.001$. Because the main analyses showed a significant effect of cognitive domain, we reran the analysis with cognitive domain as a covariate. This did not alter the effect of training type, $g=0.06[-0.05$, $0.18], p=0.300$.

We did not find any effect of the number of sessions, $Q_{M}$ (1) $=0.02, g=-0.01[-0.13,0.12], p=0.902$, and $g=-0.03$ $[-0.14,0.09], p=0.685$, with cognitive domain as a covariate. Neither did we find any effect of hours of training, $Q_{M}(1)=$ $0.03, g=0.01[-0.12,0.14], p=0.872$, and $g=g=0.00$ $[-0.12,0.12], p=0.981$ with cognitive domain as a covariate.

We then analyzed whether sample age moderated the training effect. We found no effect of age, $Q_{M}(1)=0.07, g=0.03$ $[-0.17,0.22], p=0.794$. Adding cognitive domain as a covariate to the analysis did not alter the effect, $g=0.02[-0.16$, $0.20], p=0.834$.

\section{Transfer of N-back training to different materials}

To investigate whether dual n-back training produced different effects depending on the transfer task materials (verbal vs. nonverbal), we conducted an analysis in which only WM and Gf measures were included. The n-back measures and cognitive control measures were not included, because the number of effect sizes was insufficient. The difference between verbal and nonverbal transfer measures was not significant, $Q_{M}(3)=1.77, p=0.623$. The test for residual heterogeneity was not significant, $Q_{E}(103)=123.43, p=0.08$ (Fig. 6).

\section{Active and passive control groups}

To investigate whether the pre-post change in active and passive control groups differed, we conducted a comparison between active and passive control groups within the same experiment. Here $g$ is calculated similarly to the treatment effect; the difference in $g_{\text {WITHIN }}$ between the active and the passive control group. This analysis of, in total, 34 effect sizes revealed no differences between the two types of control groups, $g=0.03[-0.07,0.12], p=0.429$. The effect of the type of control group was not significantly different between the four cognitive domains, $Q_{M}(3)=6.81, p=0.078$. 


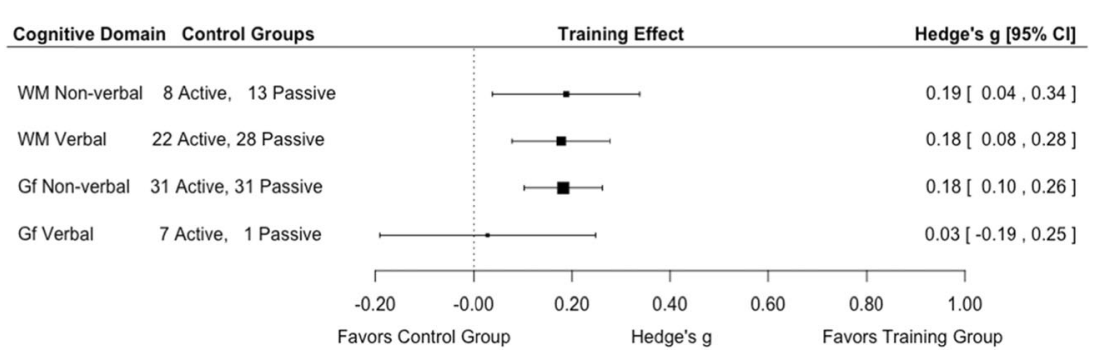

Fig. 6 For both verbal and nonverbal transfer task materials for fluid intelligence (here: reasoning) and working memory (WM), the figure displays synthesized effect sizes and confidence intervals for the

comparison of pre-post improvements between training groups and control groups. Positive values favor the training groups and negative values favor control groups.

\section{Discussion}

We examined the efficacy of one of the most commonly used WM training paradigms, namely the $n$-back task. In contrast to previous meta-analyses in the field, we did not lump untrained versions of the training task together with the other WM transfer measures. Instead, we deemed it as important to keep these two types of transfer measures separate to be able to examine whether n-back training mainly yields taskspecific transfer or more general WM improvement. We also took into account certain methodological shortcomings that limit the interpretability of previous meta-analyses on WM training effects. From each original study sample, previous meta-analyses have included only one measure (or an average of several measures) per cognitive domain in the analyses. We employed a multi-level meta-analytical approach to be able to include all measures from the original studies, and thereby obtain a less biased estimate of the training effects. We took full advantage of the repeated measures design in the original studies by accounting for the correlation between pre- and posttest performance and thereby increasing the statistical power of our analyses.

\section{Main analyses}

The present meta-analysis included 203 training effects (190 after data screening) from 33 studies. These studies consisted of 41 unique experiments. In total, data were obtained from 2,105 individuals. The results from the main analyses showed a moderate effect of task-specific transfer to untrained n-back tasks and very small transfer effects to other untrained WM measures, cognitive control, and Gf.

The transfer effect to untrained WM tasks as a whole is approximately of the same size as in the n-back training analyses by Melby-Lervåg et al. (2016; when averaged across verbal and nonverbal WM and active and passive control groups) but considerably smaller than in the other metaanalyses not focusing specifically on n-back training (MelbyLervåg \& Hulme, 2013; Weicker et al., 2016; Schwaighofer et al., 2015; Table 1). There are several possible reasons for this discrepancy. First, Melby-Lervåg \& Hulme (2013) who reported the highest effects of near transfer, in some cases included the training task in their analyses, resulting in an overestimation of the near transfer effect. Second, except for Melby-Lervåg and Hulme (2013), all previous meta-analyses investigating near transfer of WM training have excluded simple spans from the WM transfer domain. We decided to include simple spans as it has been argued that simple and complex spans (the latter ones being included both in previous metaanalyses and the present one) can in fact be considered as measures of the same cognitive processes (Unsworth \& Engle, 2007). Nevertheless, when we ran the same analysis without the simple spans, the effect size remained similar ( $g$ $=0.21$ ). Third, Jaeggi et al. (2010) have suggested that the near transfer effects may be smaller following n-back training than training with other WM training paradigms, because the nback task shows low correlations with other WM tasks. However, a recent study showed that n-back tasks are in fact highly correlated with other WM tasks at a latent level (Schmiedek et al., 2014). Fourth, the differences between the size of the near transfer effect in the previous meta-analyses and the present one may be partly related to differences in the inclusion of studies, and the fact that we included all relevant measures from each original study in our analysis. Finally, in light of the present results, perhaps the most important explanation for higher effect sizes for WM transfer in previous metaanalyses is the fact that those studies have not separated untrained variants of the training task from other WM tasks. This could be even a more acute problem in meta-analyses on studies using several training tasks where it becomes more likely that the transfer tasks include untrained versions of the training tasks. In an attempt to investigate this, we reanalyzed the data from healthy adults included in Melby-Lervåg et al. (2016) by separating between untrained versions of the training task(s) and other untrained WM tasks. We excluded those studies in which the n-back task was the only training task in order to be able to examine if the inclusion of untrained versions of the training task(s) leads to an overestimation of the near transfer effects also for other training paradigms. The pooled effect size for all types of WM tasks was $g=0.29[0.18,0.40] p<0.001$. However, when analyzing untrained versions of the training tasks separately from other WM tasks, the results showed that 
the effect size was significantly stronger, $Q_{M}(1)=8.89, p<$ 0.01 , for the former, $g=0.62[0.38,0.86] p<0.001$ (number of effect sizes $=19$ ), than the latter, $g=0.21,[0.08,0.33] p<0.01$ (number of effect sizes $=70$ ). The findings of this reanalysis are in line with the present meta-analysis and indicate that also for other WM training paradigms, task-specific transfer is important to take into account when investigating transfer effects. These results go against the idea that the transfer effects in the present study are lower because of issues with concurrent validity of the n-back task.

For cognitive control, the transfer effect was approximately of the same size as in the two previous meta-analyses investigating transfer of different kinds of WM training to executive functions and attention (Melby-Lervåg \& Hulme, 2013; Weicker et al., 2016). Also for Gf, the present transfer effect size is roughly in line with the three previous meta-analyses investigating transfer to Gf from n-back training (Au et al., 2015; Melby-Lervåg \& Hulme, 2016; Melby-Lervåg et al., 2016).

In sum, in the present meta-analysis, the only notable transfer effect is seen to untrained n-back tasks. Despite the fact that the transfer effects to the other domains are also statistically significant, they can be considered very small. This is because an effect size of 0.2 means that only approximately $1 \%$ of the variance of the dependent variable (e.g., score on a Gf task) can be explained by which group (training or control group) the participant belongs to. The practical significance of such effects can thus be questioned (see also Melby-Lervåg \& Hulme, 2016).

\section{Mechanisms behind transfer}

We hypothesized that if $n$-back training enhances the WM components it consists of, the magnitude of transfer effects would follow the presumed cognitive overlap between the transfer tasks and the training task (Dahlin et al., 2008; Waris et al., 2015). This would result in a gradual decrease in effect sizes, with the strongest transfer effects to untrained n-back tasks, followed by other WM tasks, cognitive control, and Gf. However, in the present study, the only noteworthy transfer effect was seen to untrained n-back tasks, while transfer to other tasks was at similar, very small levels. This pattern of results suggests that the transfer effects of n-back training are mainly caused by acquisition of task-specific aspects such as suitable strategies, rather than better-functioning WM components. This is because taskspecific improvement can enhance performance only on tasks with a similar structure where the same strategies can be succesfully employed. An actual improvement in the effectiveness of the underlying WM components such as flexibility of updating and storage capacity, on the other hand, should result in broad transfer effects to different kinds of measures (von Bastian \& Oberauer, 2014). A potential caveat is that a similar pattern of results as the one seen here could also be due to an improvement that is limited to the updating component. This is because we did not separate between updating tasks and other WM tasks in the analyses. However, a post hoc analysis of our data showed that the transfer effects to updating tasks other than n-back ( $g=0.26)$ were roughly of the same size as the transfer effects to other WM tasks $(g=0.23)$, strengthening the conclusion that $\mathrm{n}$-back training mainly improves task-specific aspects and not WM. It may thus be that the very small and similar effects of transfer to other WM tasks, Gf tasks and tasks measuring cognitive control reflect some general effects such as enhanced attention, perceptual speed, or getting used to the computer and performing demanding cognitive tasks.

\section{Moderator analyses}

We also investigated whether the choice of control group affected the training outcome. The results showed no differences in improvement between passive and active control groups when comparing them within studies that had employed both types of control groups. Based on these results, we agree with $\mathrm{Au}$ et al. (2015) in that there does not seem to be any clear support for the idea that Hawthorne effects affect the results. However, when adding the type of control group as a co-variate to our main analysis, the results revealed a small, significant effect. This could mean either of two things: the within-study comparison was underpowered, failing to find a true difference, or the training groups perform better in studies with passive controls than in studies with active controls. The latter explanation has also been proposed by Au et al. (2015).

Also, the results from the present meta-analysis do not give us reason to claim that publication bias plays a major role in the results. However, the reviewed studies contained a few observations that could be considered outliers. These were removed, because they either over- or underestimated the training effect beyond what was expected from sampling error alone.

Regarding the other moderators in the present meta-analysis, the results showed no difference in transfer effects between young and old participants. On the whole, this is in line with the previous meta-analyses (Melby-Lervåg \& Hulme, 2013; Schwaighofer et al., 2015; Melby-Lervåg et al., 2016). The results also showed that training with single or dual nback tasks was equally effective in producing transfer to the four cognitive domains. This concurs with the Au et al. (2015) meta-analysis showing similar transfer effects to Gf after single and dual n-back training. The number of training hours or sessions did not affect the transfer results either, again in line with most of the previous meta-analyses. However, Weicker et al. (2016) found that the number of training sessions was positively related to the size of the transfer effect to WM. This difference in results may stem from several sources. While the present meta-analysis focused on n-back training in healthy 
adults, the Weicker et al. (2016) meta-analysis included studies investigating all kinds of WM training paradigms in both healthy and clinical samples of children and adults. It is in principle also possible that higher amounts of n-back training than what is currently used might yield stronger effects. Finally, in line with the Au et al. (2015) meta-analysis, our results indicated that it did not matter if the WM and Gf transfer tasks consisted of verbal or nonverbal material.

\section{Limitations of the present study}

The present meta-analysis focused on only one type of WM training, namely n-back training. By restricting the analysis to only one training task type, it was easier to interpret the present pattern of transfer effects. Investigating transfer elicited by several training tasks makes it even more challenging to separate between task-specific effects and increased effectiveness of general WM mechanisms. We were able to conclude that out of the four cognitive domains studied here, n-back training produces substantial transfer only to untrained variants of the training task. Transfer effects to other tasks (whether they measure WM, Gf, or cognitive control), albeit being observable, are small and apparently of little practical significance.

Due to the limited number of available studies, we were not able to investigate transfer to other cognitive domains than the four studied here. For the same reason, we could not perform all the moderator analyses for all four cognitive domains (e.g., material-specific aspects of transfer were investigated only for WM and Gf), and we refrained from investigating interactions between moderators.

\section{Directions for future studies}

There is still much controversy regarding the efficacy of WM training, despite the fact that the issue has been investigated in many training studies and a few meta-analyses. We believe that much of the controversy is due to the great variability between training studies regarding for example the choice of training and transfer tasks, control group, and study population. This variability is then reflected in meta-analyses as well, because different researchers will make different choices in categorizing tasks, participant groups, and studies. On the one hand, one could think that variation in for example transfer tasks is important in order to be able to draw conclusions about improvement in general WM mechanisms (for a discussion, see e.g., Shipstead, Redick et al., 2010; Shipstead et al., 2012). This is because cognitive tasks always involve variance that stems from other sources than the ability of interest. Apart from random error (such as variation in alertness or disturbing noises in the testing environment), such variance comes from other abilities engaged in solving the tasks, strategies employed by the participants, or differences related to the type of stimuli involved. Therefore, it has been recommended that training studies should utilize factor analysis to analyze transfer at a latent variable level that should provide more reliable information about the ability of interest compared to task-specific performance (Schmiedek, Lövdén, \& Lindenberger, 2010). On the other hand, the present results indicate that task-specific aspects play an important role in the transfer effects. A failure to make a distinction between task-specific and other WM transfer not only inflates effect sizes for near transfer, but may even lead one to a wrong track when searching for theoretical explanations for WM training effects (i.e., assuming that WM training increases the effectiveness of WM in general, rather than considering also alternative hypotheses on strategy-based effects). Thus, if one wants to shed more light on the underlying mechanisms of transfer, more emphasis should be put on task-specific aspects. It would be important to systematically analyze performances in tasks that are closely related to the training task, in order to find out which are the mechanisms that drive the major transfer effects.

As previously mentioned, there is, however, not much research on what different executive tasks actually measure and how reliable they are over time. Low task reliabilities together with small sample sizes (often employed in training studies), result in weak statistical power and consequently lower chances of observing a putative effect of training.

We believe that it would be important for future studies to try to solve issues related to task reliability and validity, pairing of training and transfer tasks, and statistical power, rather than conducting more training studies that carry the current methodological problems. Ultimately, training effects should also be evaluated with measures that are more closely tapping reallife working memory demands. Furthermore, future studies should pave a way to a theory of the processes involved in a training-induced change in WM, a theory that is currently missing (Gibson, Gondoli, Johnson, Steeger, \& Morrissey, 2012). Our results highlighting the role of task-specific transfer suggest that a considerable part of the transfer effects is related to self-generated performance strategies that emerge during a repeated practice with a limited set of WM tasks. We concur with what Shipstead, Hicks, and Engle (2012) stated 4 years ago: "Working memory training remains a work in progress."

\section{Conclusions}

The present meta-analysis on the efficacy of n-back training shows medium transfer effects to untrained versions of the trained n-back tasks and small transfer effects to other WM tasks, cognitive control, and Gf. Our results suggest that previous meta-analyses investigating the effects of WM training have overestimated the transfer effects to WM by including untrained variants of the training tasks in their WM transfer domain. Consequently, transfer of n-back training is more task-specific than has previously been suggested. 
Acknowledgments The authors thank Daniel Fellman for coding the articles for the reliability assessment and Eric Karlsson for creating Fig. 1. They also thank Jacky Au, Martin Buschkuehl, Céline N. Bürki, Roberto Colom, Stephan Heinzel, Susanne M. Jaeggi, Andy C. H. Lee, Jonas Lindeløv, Vladimír Marček, Stephan Oelhafen, James G. Phillips, Thomas S. Redick, Tiina Salminen, Hillary Schwarb, Susanne Schweizer, Rachael D. Seidler, Shamus P. Smith, Barbara StuderLuethi, Clayton L. Stephenson, Oshin Vartanian, and Ludmila ZajacLamparska for providing additional information regarding their studies.

\section{References}

References marked with an asterisk indicate studies included in the meta-analysis.

*Anguera, J. A., Bernard, J. A., Jaeggi, S. M., Buschkuehl, M., Benson, B. L. Jennett, S., ...Seidler, R. D. (2012). The effects of working memory resource depletion and training on sensorimotor adaptation. Behavioral Brain Research, 228(1), 107-115. doi:10.1016/j.bbr.2011.11.040

Au, J., Buschkuehl, M., Duncan, G. J., \& Jaeggi, S. M. (2016). There is no convincing evidence that working memory training is NOT effective: A reply to Melby-Lervåg and Hulme (2015). Psychonomic Bulletin \& Review, 23(1), 331-337. doi:10.3758/s13423-015-0967-4

Au, J., Sheehan, E., Tsai, N., Duncan, G. J., Buschkuehl, M., \& Jaeggi, S. M. (2015). Improving fluid intelligence with training on working memory: A meta-analysis. Psychonomic Bulletin \& Review, 22(2), 366-377. doi:10.3758/s13423-014-0699-x

Bäckman, L., Nyberg, L., Soveri, A., Johansson, J., Andersson, M., Dahlin, E., ... Rinne, J. O. (2011). Effects of working-memory training on striatal dopamine release. Science, 333(6043), 718. doi: 10.1126/science. 1204978

Baddeley, A. (2000). The episodic buffer: A new component of working memory? Trends in Cognitive Sciences, 4(11), 417423. doi:10.1016/S1364-6613(00)01538-2

*Beavon, P. (2012). Improving memory using n-back training (Bachelor's thesis). Retrieved from http://ro.ecu.edu.au/theses hons/65/

Becker, B. J. (2000). Multi-variate meta-analysis. In H. E. A. Tinsley \& E. D. Brown (Eds.), Handbook of Applied Multivariate Statistics and Mathematical Modelling (pp. 499-525). Orlando: Academic Press.

Bishop, D. V., \& Thompson, P. A. (2016). Problems in using p-curve analysis and text-mining to detect rate of $\mathrm{p}$-hacking and evidential value. PeerJ, 4, e1715. doi:10.7717/peerj.1715

Bogg, T. \& Lasecki, L. (2014). Reliable gains? Evidence for substantially underpowered designs in studies of working memory training transfer to fluid intelligence. Frontiers in Psychology, 22(5). doi: 10.3389/fpsyg.2014.01589

Brehmer, Y., Westerberg, H., \& Bäckman, L. (2012). Workingmemory training in younger and older adults: Training gains, transfer, and maintenance. Frontiers in Human Neuroscience, 6(63). doi:10.3389/fnhum.2012.00063

*Bürki, C. N., Ludwig, C., Chicherio, C., \& De Ribaupierre, A. (2014). Individual differences in cognitive plasticity: An investigation of training curves in younger and older adults. Psychological Research, 78(6), 821-835. doi:10.1007/s00426-014-0559-3

*Buschkuehl, M., Hernandez-Garcia, L., Jaeggi, S.M., Bernard, J. A., \& Jonides, J. (2014). Neural effects of short-term training on working memory. Cognitive, Affective, \& Behavioral Neuroscience, 14(1), 147-160. doi: 10.3758/s13415-013-0244-9
*Chooi, W.-T., \& Thompson, L. A. (2012). Working memory training does not improve intelligence in healthy young adults. Intelligence, 40(6), 531-542. doi:10.1016/j.intell.2012.07.004

*Colom, R., Román, F. J., Abad, F. J., Shih, P. C., Privado, J., Froufe, M., ... Jaeggi, S.M. (2013). Adaptive n-back training does not improve fluid intelligence at the construct level: Gains on individual tests suggest that training may enhance visuospatial processing. Intelligence, 41(5), 712-727. doi:10.1016/j.intell.2013.09.002

Dahlin, E., Neely, A. S., Larsson, A., Bäckman, L., \& Nyberg, L. (2008). Transfer of learning after updating training mediated by the striatum. Science, 320(5882), 1510-1512. doi:10.1126/science.1155466

Dougherty, M. R., Hamovitz, T., \& Tidwell, J. W. (2016). Reevaluating the effectiveness of n-back training on transfer through the Bayesian lens: Support for the null. Psychonomic Bulletin \& Review, 23(1), 306-316. doi:10.3758/s13423-015-0865-9

Duval, S., \& Tweedie, R. (2000). Trim and fill: A simple funnel-plot based method of testing and adjusting for publication bias in metaanalysis. Biometrics, 56(2), 455-463.

Egger, M., Smith, G. D., Schneider, M., \& Minder, C. (1997). Bias in meta-analysis detected by a simple, graphical test. BMJ, 315(7109), 629-634. doi:10.1136/bmj.315.7109.629

Engle, R. W. (2002). Working memory capacity as executive attention. Current Directions in Psychological Science, 11(1), 19-23.

Gibson, B. S., Gondoli, D. M., Johnson, A. C., Steeger, C. M., \& Morrissey, R. M. (2012). The future promise of Cogmed working memory training. Journal of Applied Research in Memory and Cognition, 1(3), 214-216. doi:10.1016/j.jarmac.2012.07.003

Green, C. S., \& Bavelier, D. (2008). Exercising your brain: A review of human brain plasticity and training-induced learning. Psychology and Aging, 23(4), 692-701. doi:10.1037/a0014345

*Heffernan, A. E. (2014). The generalizability of dual n-back training in younger adults (Master's thesis). Retrieved from http://hdl.handle. net/10222/56021

*Heinzel, S., Lorenz, R. C., Pelz, P., Heinz, A., Walter, H., Kathmann, N., ... Stelzel, C. (2016). Neural correlates of training and transfer effects in working memory in older adults. NeuroImage, 134, 236249. doi: 10.1016/j.neuroimage.2016.03.068

*Heinzel, S., Schulte, S., Onken, J., Duong, Q-L., Riemer, T. G., Heinz, A., ... Rapp, M. A. (2014). Working memory training improvements and gains in non-trained cognitive tasks in young and older adults. Aging, Neuropsychology, and Cognition, 21(2), 146-173. doi: $10.1080 / 13825585.2013 .790338$

Hindin, S. B., \& Zelinski, E. M. (2012). Extended practice and aerobic exercise interventions benefit untrained cognitive outcomes in older adults: A meta-analysis. Journal of the American Geriatrics Society, 60(1), 136-141. doi:10.1111/j.1532-5415.2011.03761.x

*Jaeggi, S. M., Buschkuehl, M., Jonides, J., \& Perrig, W. J. (2008). Improving fluid intelligence with training on working memory. PNAS, 105(19), 6829-6833. doi:10.1073/pnas.0801268105

*Jaeggi, S. M., Buschkuehl, M., Shah, P., \& Jonides, J. (2014). The role of individual differences in cognitive training and transfer. Memory and Cognition, 42(3), 464-480. doi:10.3758/s13421-013-0364-z

*Jaeggi, S. M., Buschkuehl, M., \& Jonides, J. (2009). Working memory training and transfer. Unpublished data presented at the ONR contractor's meeting, Arlington, VA.

*Jaeggi, S. M., Studer-Luethi, B., Buschkuehl, M., Su, Y.-F., Jonides, J., \& Perrig, W. J. (2010). The relationship between n-back performance and matrix reasoning - implications for training and transfer. Intelligence 38(6), 625-635. doi:10.1016/j.intell.2010.09.001

*Kane, M. J., Hambrick, D. Z., \& Conway, A. R. A. (2005). Working memory capacity and fluid intelligence are strongly related constructs: Comment on Ackerman, Beier, and Boyle (2005). Psychological Bulletin, 131(1), 66-71. doi:10.1037/00332909.131.1.66. author reply 72-75.

Karbach, J., \& Verhaeghen, P. (2014). Making working memory work: A meta-analysis of executive-control and working memory training in 
older adults. Psychological Science, 25(11), 2027-2037. doi:10.1177/0956797614548725

Karr, J. E., Areshenkoff, C. N., Rast, P., \& Garcia-Barrera, M. A. (2014). An empirical comparison of the therapeutic benefits of physical exercise and cognitive training on the executive functions of older adults: A meta-analysis of controlled trials. Neuropsychology, 28(6), 829-845. doi:10.1037/neu0000101

Kelly, M. E., Loughrey, D., Lawlor, B. A., Robertson, I. H., Walsh, C., \& Brennan, S. (2014). The impact of cognitive training and mental stimulation on cognitive and everyday functioning of healthy older adults: A systematic review and meta-analysis. Ageing Research Reviews, 15, 28-43. doi:10.1016/j.arr.2014.02.004

Kirchner, W. K. (1958). Age differences in short-term retention of rapidly changing information. Journal of Experimental Psychology, 55(4), $352-358$.

Klingberg, T. (2010). Training and plasticity of working memory. Trends in Cognitive Sciences, 14(7), 317-324. doi:10.1016/j.tics.2010.05.002

Klingberg, T., Fernell, E., Olesen, P. J., Johnson, M., Gustafsson, P., Dahlström, K., ... Westerberg, H. (2005). Computerized training of working memory in children with ADHD-a randomized, controlled trial. Journal of the American Academy of Child and Adolescent Psychiatry, 44(2), 177-186.

Klingberg, T., Forssberg, H., \& Westerberg, H. (2002). Training of working memory in children with ADHD. Journal of Clinical and Experimental Neuropsychology, 24(6), 781-791.

Kundu, B., Sutterer, D. W., Emrich, S. M., \& Postle, B. R. (2013). Strengthened effective connectivity underlies transfer of working memory training to tests of short-term memory and attention. The Journal of Neuroscience, 33(29), 8705-8715. doi:10.1523/JNEUROSCI.5565-12.2013

*Küper, K., \& Karbach, J. (2016). Increased training complexity reduces the effectiveness of brief working memory training: Evidence from short-term single and dual n-back training interventions. Journal of Cognitive Psychology, 28(2), 199-208. doi: 10.1080/20445911.2015.1118106

Lezak, M. D., Howieson, D. B., \& Loring, D. W. (2004). Neuropsychological Assessment. New York: Oxford University Press.

*Lilienthal, L., Tamez, E., Shelton, J. T., Myerson, J., \& Hale, S. (2013). Dual n-back training increases the capacity of the focus of attention. Psychonomic Bulletin and Review, 20(1), 135-141. doi:10.3758/s13423-012-0335-6

Lövdén, M., Bäckman, L., Lindenberger, U., Schaefer, S., \& Schmiedek, F. (2010). A theoretical framework for the study of adult cognitive plasticity. Psychological Bulletin, 136(4), 659-676. doi: $10.1037 / \mathrm{a} 0020080$

Melby-Lervåg, M., \& Hulme, C. (2013). Is working memory training effective? A meta-analytic review. Developmental Psychology, 49(2), 270-291. doi:10.1037/a0028228

Melby-Lervåg, M., \& Hulme, C. (2016). There is no convincing evidence that working memory training is effective: A reply to Au et al. (2014) and Karbach and Verhaeghen (2014). Psychonomic Bulletin \& Review, 23(1), 324-330. doi:10.3758/s13423-0150862-z

Melby-Lervåg, M., Redick, T., \& Hulme, C. (2016). Working memory training does not improve performance on measures of intelligence or other measures of "far transfer": Evidence from a meta-analytic review. Perspectives on Psychological Science, 11(4), 512-534. doi:10.1177/1745691616635612

Miller, K. M., Price, C. C., Okun, M. S., Montijo, H., \& Bowers, D. (2009). Is the n-back task a valid neuropsychological measure for assessing working memory? Archives of Clinical Neuropsychology, 24(7), 711-717. doi:10.1093/arclin/acp063

Miyake, A., Friedman, N. P., Emerson, M. J., Witzki, A. H., Howerter, A., \& Wager, T. D. (2000). The unity and diversity of executive functions and their contributions to complex "frontal lobe" tasks: A latent variable analysis. Cognitve Psychology, 41(1), 49-100.

Moreno, S. G., Sutton, A. J., Ades, A. E., Stanley, T. D., Abrams, K. R., Peters, J. L., \& Cooper, N. J. (2009). Assessment of regressionbased methods to adjust for publication bias through a comprehensive simulation study. BMC Medical Research Methodology, 9(2). doi: 10.1186/1471-2288-9-2

Morrison, A. B., \& Chein, J. M. (2011). Does working memory training work? The promise and challenges of enhancing cognition by training working memory. Psychonomic Bulletin and Review, 18(1), 4660. doi:10.3758/s13423-010-0034-0

NIMH Research Domain Criteria Workshop (2010). Working memory: Workshop proceedings. Downloaded November 8, 2016 from https://www.nimh.nih.gov/research-priorities/rdoc/workingmemory-workshop-proceedings.shtml

*Oelhafen, S., Nikolaidis, A., Padovani, T., Blaser, D., Koenig, T., \& Perrig, W. J. (2013). Increased parietal activity after training of interference control. Neuropsychologia, 51(13), 2781-90. doi:10.1016/j.neuropsychologia.2013.08.012

Peters, J. L., Sutton, A. J., Jobes, D. R., Abrams, K. R., \& Rushton, L. (2008). Contour-enhanced meta-analysis funnel plots help distinguish publication bias from other causes of asymmetry. Journal of Clinical Epidemiology, 31(10), 991-996. doi:10.1016/j. jclinepi.2007.11.010

*Prandl, A. (2012). Visual memory improvement in recognition (Bachelor's thesis). Retrieved from http://ro.ecu.edu.au/theses hons/59/

*Preece, D. (2011). The effect of working memory (n-back) training on fluid intelligence (Bachelor's thesis). Retrieved from http://ro.ecu. edu.au/theses_hons/54/

R Core Team. (2008). R: A Language and Environment for Statistical Computing. Vienna: R Foundation for Statistical Computing.

Redick, T. S., \& Lindsey, D. R. (2013). Complex span and n-back measures of working memory: A meta-analysis. Psychonomic Bulletin \& Review, 20(6), 1102-1113. doi:10.3758/s13423-013-0453-9

*Redick, T. S., Shipstead, Z., Harrison, T. L., Hicks, K. L., Fried, D. E., Hambrick, D. Z., ... Engle, R. W. (2013). No evidence of intelligence improvement after working memory training: A randomized, placebo-controlled study. Journal of Exprimental Psychology: General, 142(2), 359-379. doi:10.1037/a0029082

Rothstein, H. R., Sutton, A. J., \& Borenstein, M. (2005). Publication Bias in Meta-Analysis: Prevention, Assessment and Adjustments. Chichester: John Wiley \& Sons.

*Rudebeck, S. R., Bor, D., Ormond, A., O'Reilly, J. X. \& Lee, A. C. H. (2012). A potential spatial working memory training task to improve both episodic memory and fluid intelligence. PLoS ONE, 7(11): e50431. doi:10.1371/journal.pone.0050431

*Salminen, T., Frensch, P., Strobach, T., \& Schubert, T. (2016). Agespecific differeneces of dual n-back training. Aging, Neurospychology, and Cognition, 23(1), 18-39. doi: 10.1080 /13825585.2015.1031723

*Salminen, T., Strobach, T., \& Schubert, T. (2012). On the impacts of working memory training on executive functioning. Frontiers in Human Neuroscience, 6, 166. doi:10.3389/fnhum.2012.00166

Schmiedek, F., Lövdén, M., \& Lindenberger, U. (2010). Hundred days of cognitive training enhance broad cognitive abilities in adulthood: Findings from the COGITO study. Frontiers in Aging Neuroscience, 2, 27. doi:10.3389/fnagi.2010.00027

Schmiedek, F., Lövdén, M., \& Lindenberger, U. (2014). A task is a task is a task: Putting complex span, n-back, and other working memory indicators in psychometric context. Frontiers in Psychology, 5. doi: 10.3389/fpsyg.2014.01475

Schwaighofer, M., Fischer, F., \& Bühner, M. (2015). Does working memory training transfer? A meta-analysis including training conditions as moderators. Educational Psychologist, 50(2), 138-166. doi:10.1080/00461520.2015.1036274 
*Schwarb, H., Nail, J., \& Schumacher, E. H. (2015). Working memory training improves visual short-term memory capacity. Psychological Research, 80(1), 128-148. doi: 10.1007/s00426-015-0648-y

*Schweizer, S., Hampshire, A., \& Dalgleish, T. (2011). Extending braintraining to the affective domain: Increasing cognitive and affective executive control through emotional working memory training. PLoS ONE, 6(9), 1-5. doi:10.1371/journal.pone.0024372

Seidler, R. D., Bernard, J. A., Buschkuehl, M., Jaeggi, S., Jonides, J., \& Humfleet, J. (2010). Cognitive training as an intervention to improve driving ability in the older adult. Technical Report No. MCASTL 2010-01. Ann Arbor: University of Michigan.

Shipstead, Z., Hicks, K. L., \& Engle, R. W. (2012). Working memory training remains a work in progress. Journal of Applied Research in Memory and Cognition, 1(3), 217-219. doi:10.1016/j. jarmac.2012.07.009

Shipstead, Z., Redick, T. S., \& Engle, R. W. (2010). Does working memory training generalize? Psychologica Belgica, 50(3 \& 4), 245-276.

Shipstead, Z., Redick, T. S., \& Engle, R. W. (2012). Is working memory training effective? Psychological Bulletin, 138(4), 628-654. doi:10.1037/a0027473

Simonsohn, U., Nelson, L. D., \& Simmons, J. P. (2014). p-curve and effect size. Correcting for publication bias using only significant results. Perspectives on Psychological Science, 9(6), 666-681. doi:10.1177/1745691614553988

*Smith, S. P., Stibric, M., \& Smithson, D. (2013). Exploring the effectiveness of commercial and custom-built games for cognitive training. Computers in Human Behavior, 29(6), 2388-2393. doi:10.1016/j.chb.2013.05.014

Stanley, T. D., \& Doucouliagos, H. (2014). Meta-regression approximations to reduce publication selection bias. Research Synthesis Methods, 5(1), 60-78. doi:10.1002/jrsm.1095

*Stepankova, H., Lukavsky, J., Buschkuehl, M., Kopecek, M., Ripova, D., \& Jaeggi, S. M. (2013). The malleability of working memory and visuospatial skills: A randomized controlled study in older adults. Developmental Psychology, 50(4), 1049-1059. doi: 10.1037/a0034913

*Stephenson, C. L., \& Halpern, D. F. (2013). Improved matrix reasoning is limited to training on tasks with a visuospatial component. Intelligence, 4(5), 341-357. doi:10.1016/j.intell.2013.05.006

Szmalec, A., Verbruggen, F., Vandierendonck, A., \& Kemps, E. (2011). Control of interference during working memory updating. Journal of Experimental Psychology Human Perception \& Performance, 37(1), 137-151. doi:10.1037/a0020365

*Thompson, T. W., Waskom, M. L., Garel, K.-L. A., Cardenas-Iniguez, C., Reynolds, G. O., Winter, R., ... Gabrieli, J. D. E. (2013). Failure of working memory training to enhance cognition or intelligence. PloS ONE, 8(5). doi:10.1371/journal.pone.0063614

Unsworth, N., \& Engle, R. W. (2007). On the division of short-term and working memory: An examination of simple and complex span and their relation to higher order abilities. Psychological Bulletin, 133(6), 1038-1066. doi: 10.1037/0033-2909.133.6.1038

*Urbánek, T., \& Marček, V. (2015). Investigating the effectiveness of working memory training in the context of Personality Systems Interaction theory. Psychological Research, 80(5), 877-888, doi: 10.1007/s00426-015-0687-4

van den Noortgate, W., López-López, J. A., Marín-Martínez, F., \& Sánchez-Meca, J. (2013). Three-level meta-analysis of dependent effect sizes. Behavior Research Methods, 45(2), 576-594. doi:10.3758/s13428-012-0261-6

*Vartanian, O., Jobidon, M.-E., Bouak, F., Nakashima, A., Smith, I., Lam, Q., \& Cheung, B. (2013). Working memory training is associated with lower prefrontal cortex activation in a divergent thinking task. Neuroscience, 236, 186-194. doi: 10.1016/j.neuroscience.2012.12.060

Viechtbauer, W. (2010). Conducting meta-analyses in R with the metafor package. Journal of Statistical Software, 36(3), 1-48.

Von Bastian, C. C., \& Oberauer, K. (2014). Effects and mechanisms of working memory training: A review. Psychological Research, 78(6), 803-820. doi:10.1007/s00426-013-0524-6

Waris, O., Soveri; A., \& Laine, M. (2015). Transfer after working memory updating training. PLOS ONE, 10(9). doi:10.1371/journal. pone. 0138734

Weicker, J., Villringer, A., \& Thöne-Otto, A. (2016). Can impaired working memory functioning be improved by training? A meta-analysis with a special focus on brain injured patients. Neuropsychology, 30(2), 190-212. doi:10.1037/neu0000227

*Zajac-Lamparska, L., \& Trempala, J. (2016). Effects of working memory and attentional control training and their transfer onto fluid intelligence in early and late adulthood. Health Psychology Report, 4(1), 41-53. doi: 10.5114/hpr.2016.56846

*Zhang, Q., Buschkuehl, M., Bernat, E., \& Jaeggi, S. M. (2014, May 2225). EEG power changes as a function of working memory training. Poster presented at the 26th annual convention of the Association for Psychological Science, San Francisco, CA. 